\title{
Proteomic profiling to identify potential biomarkers of alpha-particle radiation exposure in human lung epithelial cells
}

\author{
V. Chauhan, M. Howland, S. O'hara, L.A. Beaton, T.A. Burn, \\ T.J. Stocki and R.C. Wilkins \\ Health Canada, Environmental and Radiation Health Sciences Directorate, \\ Ottawa, Ontario, K1A-1C1 \\ e-mail: Vinita_chauhan@hc-sc.gc.ca
}

\begin{abstract}
Of the radiation types, alpha- $(\alpha)$ particles are of particular interest as they are an environmental concern, predominately due to inhalation of radon and its daughter progeny. Furthermore, $\alpha$-particle emitters like Americium-241, Plutonium-238 and Polonium-210 have been identified as probable isotopes to be used in radiological dispersal devices. Thus, the identification of potential biomarkers to $\alpha$-particle radiation exposure would be useful for the development of field deployable bioassays which could be used for human risk assessment and public health protection. Human lung cells were exposed to $\alpha$-particle radiation and assessed for modulations in protein expression using two-dimensional gel electrophoresis (2D-GE). Concurrently, cell culture supernatants were analyzed for cytokine secretion using a multiplex-27 bead array assay. Cell culture supernatants assessed for cytokine secretion expressed 8 statistically significant cytokines following $\alpha$-particle exposure, among which VEGF was confirmed to be dose-responsive and not modulated in X-irradiated cells. Analysis of whole cell lysates using 2-D gel electrophoresis showed 15 upregulated and 1 downregulated protein spot, of which 4 were identified by mass spectrometry. These data suggest that $\alpha$-particle exposure results in the alterations in expression-levels of specific proteins which may be potential biomarkers used further for the development of fast and reliable bioassays.
\end{abstract}

\section{INTRODUCTION}

Counter-terrorism and national security have become high priority issues worldwide. In spite of the enormity of the magnitude of threats posed by radiological dispersal devises (RDDs), capabilities for effectively responding to such a threat are limited. We only have a vague understanding of the long-term biological effects and public health consequences of exposure to such RDDs. Alpha $(\alpha)$ particle emitters like Polonium-210, Americium-241, Plutonium-238 have been identified as probable isotopes to be used in such a weapon, as they have suitably long half-lives resulting in low continuous doses (U.S. Department of Energy http://www.evs.anl.gov/pub/dsp_detail.cfm?PubID= 1472, accessed April 2011). Most of these isotopes are present in commercial radioactive sources, in nuclear reactors or in the environment (radium-226). In 2006, Alexander Litvinenko was poisoned with ${ }^{210} \mathrm{Po}$ which emits $5.298 \mathrm{MeV} \alpha$-particles. He displayed symptoms of acute radiation syndrome (ARS) and died within a span of a week before the cause of his illness could be identified [1]. In this case, a rapid means for identifying the source of his illness could have prevented his untimely death.

Biomarkers can be defined as a biological response to an external stimulus, which differs significantly from the normal state. To date, numerous studies have mined for signature biomarkers to gamma and $\mathrm{X}$-irradiation. However, $\alpha$-particle radiation may elicit biological responses resulting in the expression of a unique subset of biomarkers due to its different mode of action. A critical characteristic of $\alpha$-particles is their high linear energy transfer (LET) values, which are typically $166 \mathrm{keV} / \mu \mathrm{m}$ for $2.5 \mathrm{MeV} \alpha$-particles in comparison to $2.0 \mathrm{keV} / \mu \mathrm{m}$ for X-rays [2]. High LET $\alpha$ tparticles create very dense ionizing tracks as they traverse a medium. Therefore, they produce more 
significant biological effects when compared to equivalent doses from low LET radiation, which are more sparsely ionizing [2]. If ingested or inhaled, $\alpha$-particles emitting radionuclides can cause significant damage to sensitive lining of cells and internal human tissue [2]. A number of in vitro and in vivo studies have shown that $\alpha$-particle exposure can lead to mutagenic changes including large deletions, frameshifts and base-change mutations (reviewed in [3]). The adverse biological effects seen after $\alpha$-particle exposure may lead to systemic effects and the expression of a wide-spectrum of biomolecules (genes, proteins, lipids and secondary messengers). These biomolecules may be differentially regulated and therefore serve as potential biomarkers and high throughput strategies and platforms are required for their screening and analysis. Therefore, the focus of this study was to identify potential biomarkers of $\alpha$-particle radiation exposure by using state-of-art complementary proteomic/Bio-Plex technology in conjunction with bioinformatics. This in turn will contribute to the development of tools for rapid and delayed incidence assessment and also provide potential mechanistic insight into the adverse health effects associated with exposure to $\alpha$-particle radiation.

\section{MATERIALS AND METHODS}

\section{Cell exposure and harvesting}

Human derived lung epithelial cells (A549) obtained from the American Type Culture Collection (ATCC, Manassas, VA, USA), were maintained in a humidified incubator at $37^{\circ} \mathrm{C}, 5 \% \mathrm{CO}_{2}, 95 \%$ air in $75 \mathrm{~cm}^{2}$ flasks (T-75). The cells were grown to confluence for 2-3 days in F-12K medium, containing $10 \%$ fetal bovine serum (ATCC) and then seeded into $35 \mathrm{~mm}$ culture dishes containing 2 layers of $2.5 \mu$ m-thick mylar films. The seeding density was at $1 \times 10^{6}$ cells/dish with $2 \mathrm{~mL}$ of culture media containing $100 \mathrm{units} / \mathrm{mL}$ of penicillin and $100 \mu \mathrm{g} / \mathrm{mL}$ streptomycin. Cells were cultured to about $90 \%$ confluency, then exposed to $\alpha$-particle radiation at doses of $0 \mathrm{~Gy}-1.5 \mathrm{~Gy}$ using ${ }^{241} \mathrm{Am}$ electroplated discs having an activity level of $66.0 \mathrm{kBq} \pm 3 \%$ (dose rate of $0.98 \pm 0.01 \mathrm{~Gy} / \mathrm{h}, \mathrm{LET}$ of $127.4 \pm 0.4 \mathrm{keV} / \mu \mathrm{m}$ ). The absorbed dose of $\alpha$-radiation to which cells were exposed was calculated using the GEANT4 v.9.1 Monte Carlo toolkit [4] For the $\alpha$-particle exposures, cells were cultured in thin Mylar based plastic dishes (MD) (Chemplex Industries, Palm City, FL, USA), which allowed penetration of the $\alpha$-particles. Cells destined for X-radiation at doses of $0 \mathrm{~Gy}-1.5 \mathrm{~Gy}$ were exposed using the X-RAD 320 X-ray irradiation system at a dose rate of $0.98 \pm 0.05 \mathrm{~Gy} / \mathrm{h}, 120 \mathrm{keV}$ (Precision X-ray, Inc.).

\section{2-D GE}

Twenty-four hours after exposure, control and irradiated cells were washed and resuspended in lysis buffer (8 M urea, 2\% 3-[(3-cholamidopropyl) dimethylammonio]-1-propanesulfonate [CHAPS], $50 \mathrm{mM}$ dithiothreitol [DTT], 0.2\% Biolyte 3/10) and subjected to initial protein clean-up using a 2D-clean-up protocol (Bio-Rad) following manufactures instructions. Aliquots $(185 \mu \mathrm{l})$ of supernatants $(140 \mu \mathrm{g}$ of protein) were loaded onto IPG strips ( $\mathrm{pH} 5-8$, Bio-Rad) and separated by isoelectric focusing $(250 \mathrm{~V}$ [20min], linear ramp to $8000 \mathrm{~V}$ [140min], rapid ramp to 40,000 V). The IPG strips was loaded onto $12 \%$ sodium dodecyl sulfate (SDS)-polyacrylamide gels (4\% stacking gels), and electrophoresis was carried out at $200 \mathrm{~V}$ for $65 \mathrm{~min}$. Gels were stained overnight (BioSafe Coomassie blue, BioRad), rinsed, and scanned using the GS-800 densitometer (Bio-Rad). Visualization of protein spots separated by the 2D gels was done using PDQuest software Version 7.1.0 (Bio-Rad Laboratories, Hercules). Protein maps were created using the PDQuest software. At each exposure condition, a master (reference) gel was created. The consensus expression of the radiation-exposed replicate group ( 3 gels) was compared to that of the control replicate group ( 3 gels), spot by spot, after normalization of the density of each scanned spot to a reference to correct for slight differences in loading. Statistically significant proteins were excised using the ProXCISIONTM Proteomics Gel Cutting Robot. Proteins were in gel digested on the MassPrep Workstation.Peptide extract and analyzed by LC-MS/MS on a Nanopump series 1000 (Agilent Technologies, Mississauga, ON) coupled to a Q-TOF microTM (Waters) equipped with a 
Nanosource modified with a nanospray adapter using standard procedures. The peak lists generated was searched using the Mascot search algorithm against the MSDB database at $50 \mathrm{ppm}$ mass accuracy with the species restricted to human.

\section{Bio-Plex Suspension Bead Array assay}

Twenty-four and four hours following exposures, supernatants from exposed (1.5 Gy $\alpha$-particle) and control samples were analyzed for secretion levels of interleukin (IL)-2, IL-4, IL-5, IL-6, IL-10, IL-12, IL-13, IL-17, interferon- $\gamma$ (IFN- $\gamma$ ), granulocyte/macrophage colony-stimulating factor (GMCSF), eotaxin, fibroblast growth factor (FGF), interferon gamma-induced protein $10 \mathrm{kDa}$ (IP-10), monocyte chemotactic protein-1 (MCP-1), macrophage inflammatory protein (MIP)-1a, platelet derived growth factor (PDGF), MIP, regulated upon activation, normal t-cell expressed, and secreted (RANTES), vascular endothelial growth factor (VEGF), and tumour necrosis factor- $\alpha$ (TNF- $\alpha$ ), using a multiplex assay as prepared according to the manufacturer's instructions (Bio-Rad). Briefly, conjugated beads were allowed to react with a sample containing a known (standard) or unknown amount of cytokines for thirty minutes. Conjugated beads with bound target were then washed and incubated with biotinylated detection antibodies that were directed against specific cytokine epitopes. The resulting complexes were then incubated for a further $10 \mathrm{~min}$ with streptavidin-phycoerythrin and excess reagent was washed off and assessed for bound cytokine using a microtiter plate reader (Bio-Rad). The concentration of cytokines in supernatants was then assessed from the generated standard curves for each of individual cytokine.

\section{VEGF enzyme linked immunoassay (ELISA)}

Validation of significantly expressing proteins that were obtained using Bio-Plex technology was conducted using an immunoassay. For this study, only VEGF was further validated at three doses (0, 0.5 Gy, 1.0 Gy and 1.5 Gy) using VEGF ELISA kit (Invitrogen) as per manufactures instructions.

\section{Statistical analysis}

For ELISA measurements, statistical differences $(\mathrm{p}<0.05)$ between treatment groups and controls were determined by a repeated-measures design one-way analysis of variance (ANOVA) with Dunnett's multiple comparisons post-hoc testing using GraphPad InStat version 3.00 for Windows 95 (San Diego California USA, www . graphpad.com). Analysis was based on an $n=4$ sample size. For the Bio-Plex samples and 2D-gel electrophoresis data, statistical differences $(\mathrm{p}<0.05)$ between control and exposed treatment groups was determines using a student-T-test and was based on an $n$ sample size of $n=5$ and $=3$ respectively.

\section{RESULTS}

\section{D-GE}

Global alterations in the expression of intracellular proteome was assessed following exposure of human lung epithelial cells to $1.5 \mathrm{~Gy}$ of $\alpha$-particle radiation using 2D-GE coupled with mass spectrometry. Twenty-four hours post-exposure total cell lysates were extracted and subjected to 2D-GE. Gel images analyzed by PDQuest software were shown to express 15 statistically significant protein spots. Four of these spots were identified by mass spectrometry (Figure 1). These four proteins were shown to be upregulated ( $\geq 2$ fold) relative to the untreated control samples and included Annexin A2, Peroxiredoxin-6, Proteosome subunit $\alpha$ type-3, and heat shock protein A8. 


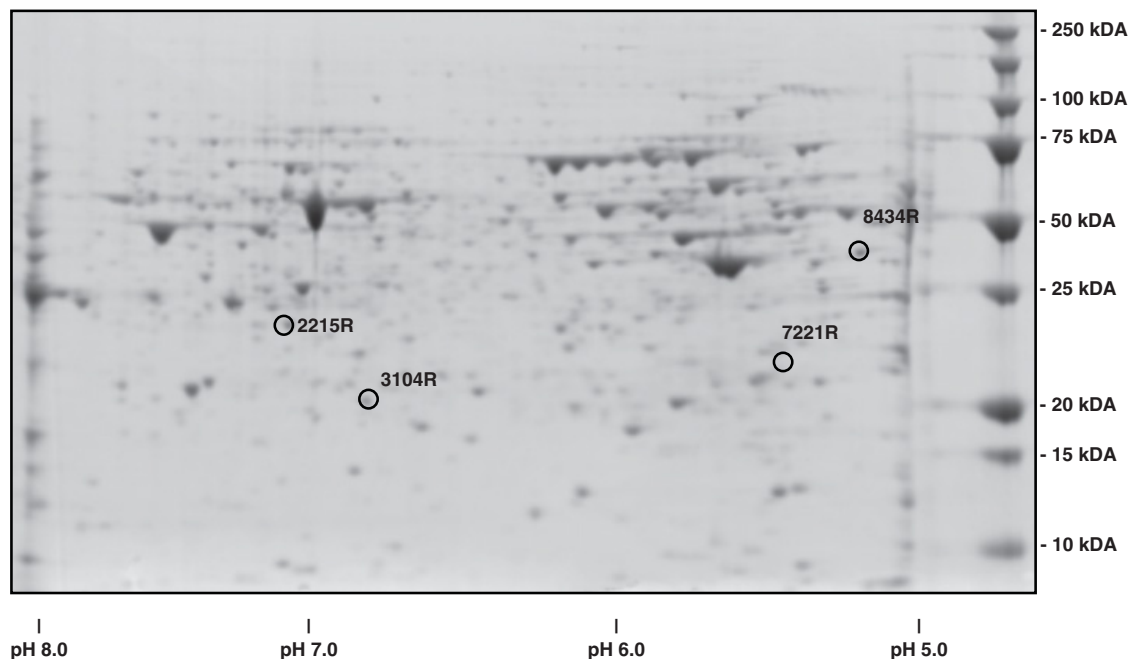

\begin{tabular}{|c|c|c|c|c|c|c|c|}
\hline $\begin{array}{c}\text { Spot } \\
\text { Number }\end{array}$ & Protein & $\begin{array}{c}\text { MASCOT } \\
\text { Score }\end{array}$ & $\begin{array}{c}\text { \# of } \\
\text { Peptides } \\
\text { Matched }\end{array}$ & $\begin{array}{c}\text { Sequence } \\
\text { Coverage } \\
(\%)\end{array}$ & $\begin{array}{c}\text { MW } \\
\text { (Da) }\end{array}$ & pl & $\begin{array}{c}>95 \\
\text { Cl }\end{array}$ \\
\hline 2215 & ANXA2 & 123 & 8 of 9 & 21 & 38677 & 7.56 & Y \\
\hline 3104 & PRDX6_HUMAN & 82 & 5 of 7 & 21 & 25002 & 6.02 & Y \\
\hline 7221 & PSA3_HUMAN & 56 & 6 of 18 & 20 & 28512 & 5.19 & N \\
\hline 8434 & Q96IS6_HUMAN & 108 & 10 of 14 & 14 & 64804 & 5.36 & Y \\
\hline
\end{tabular}

Figure 1. Protein ID using mass spectrometry of spots shown to be up-regulated by two fold or greater in lung epithelial cells exposed to $1.5 \mathrm{~Gy} \alpha$-particle radiation. $140 \mu \mathrm{g}$ of protein from each fraction was loaded onto $11 \mathrm{~cm}$ IPG strips (Bio-Rad) in PI ranges 5-8. Gels were stained overnight with Coomassie Brilliant Blue and imaged using a densitometer. Circled spots depict proteins that are up-regulated ( $\geq 2$ fold) $24 \mathrm{~h}$ after exposure to $1.5 \mathrm{~Gy}$ $\alpha$-particles using PDquest software (Bio-Rad). MW, molecular weight; PI, isolectric point., CI confidence interval.

\section{Bio-plex assay}

Secreted proteins from cell cultures exposed to radiation were assessed using multi-plex bead array technology. Twenty four hours post-exposure, antibodies to a focussed panel of 27 human inflammatory cytokines were incubated with media from cells exposed to either $\alpha$-particle radiation or X-rays. Of the 27 cytokines tested only 8 were detectable in the culture media of A549 cells (Figure 2), the remaining were below the threshold of detection. Of these 8 cytokines, 7 were downregulated (Figure 2) and only one was upregulated. It was found that VEGF had relatively strong expression levels $\sim 6 * 10^{-3}(\mathrm{pg} / \mathrm{ml}) /$ cell and was highly significant $(\mathrm{p}>0.001)$. A comparison of the response to $1.5 \mathrm{~Gy}$ of $\mathrm{X}$-irradiated cells showed a significant downregulation in the levels of 3 cytokines (Figure 3 ). Of these three cytokines both eotaxin and IFN- $\gamma$ were shown to be common to the $\alpha$-particle irradiated samples.

\section{Enzyme linked immuno-sorbent assay}

An ELISA was performed to validate cytokines that were shown to be strong expressors using Bio-Plex technology. Only one cytokine, VEGF, was shown to be significantly modulated following exposure to $1.5 \mathrm{~Gy}$ of radiation and not after X-ray exposure. This response was further validated at lower doses of radiation. Analysis of the data showed VEGF secretion to be dose-responsive and statistically 

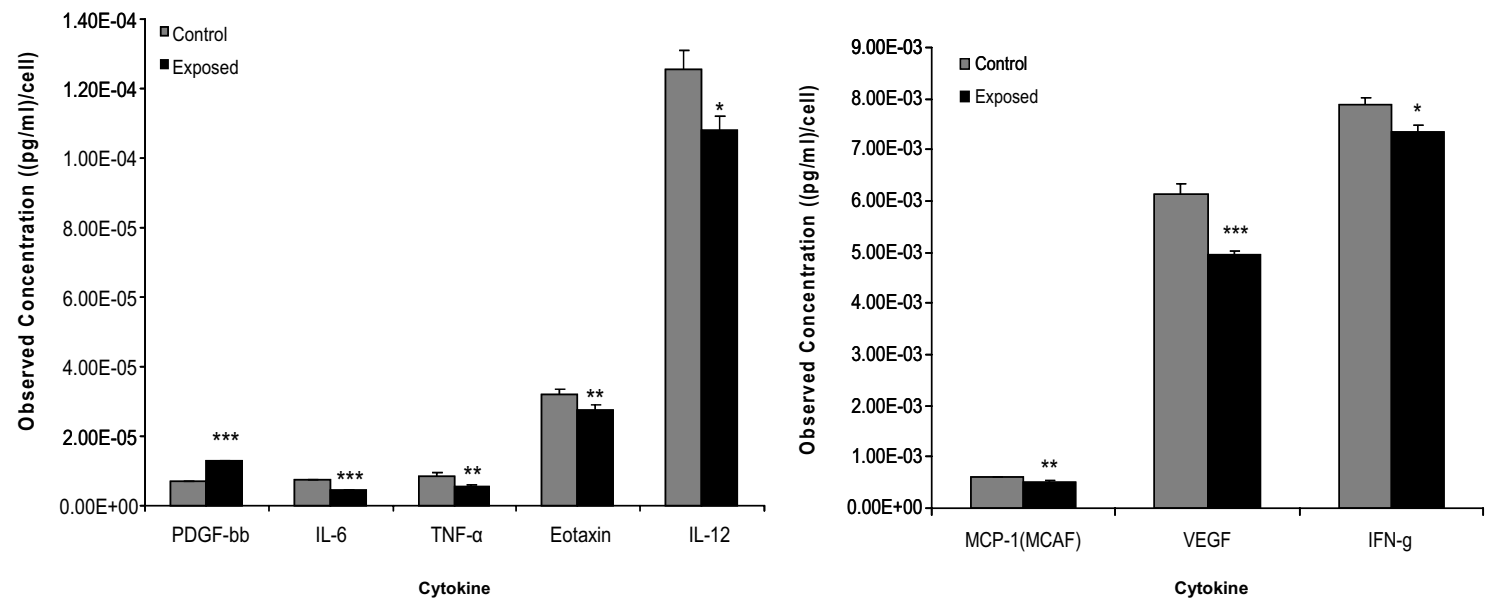

Figure 2. Cell culture supernatants from $\alpha$-particle exposed samples were treated with protease inhibitor and analyzed using Bio-Plex technology for the expression of 27 pro-inflammatory cytokines. Results showing statistical significance are presented. ${ }^{*}$ represents $\mathrm{p}<0.05,{ }^{* *}$ represents $\mathrm{p}<0.01$, and ${ }^{* * *}$ represents $\mathrm{p}<0.001$, $\mathrm{n}=5$ biological replicates. Bars are plotted as means \pm SEM.
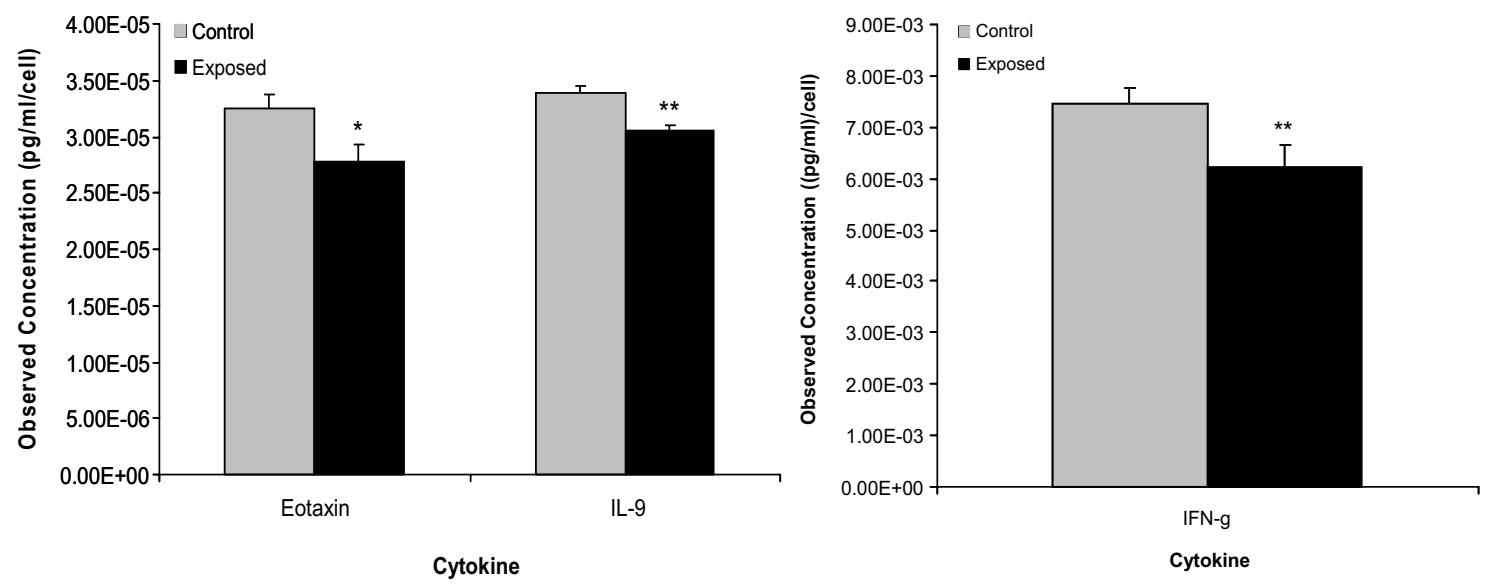

Figure 3. Cell culture supernatants from X-ray exposed samples were treated with protease inhibitor and analyzed using Bio-Plex technology for the expression of 27 pro-inflammatory cytokines. Results showing statistical significance are presented. ${ }^{*}$ represents $\mathrm{p}<0.05$ and ${ }^{* *}$ represents $\mathrm{p}<0.01, \mathrm{n}=5$ biological replicates. Bars are plotted as means \pm SEM.

significant at all three doses tested in cells exposed to $\alpha$-particle radiation. There was a highly significant $(\mathrm{p} \leq 0.001)$ linear trend $\left(\mathrm{R}^{2}=0.9721\right)$ In contrast, $\mathrm{X}$-irradiated cells did not express statistically significant levels of VEGF at similar doses tested (Figure 4).

\section{DISCUSSION}

Differential proteome analysis of a human epithelial cell-line exposed to $\alpha$-particle radiation showed a selective few proteins to be quantitatively changed between unexposed and exposed samples. On average, a total of $\sim 1800$ spots were identified consistently between control and treatment groups. Of these spots, only a small portion $(<1 \%)$ were considered differentially expressed (Figure 1). Although 


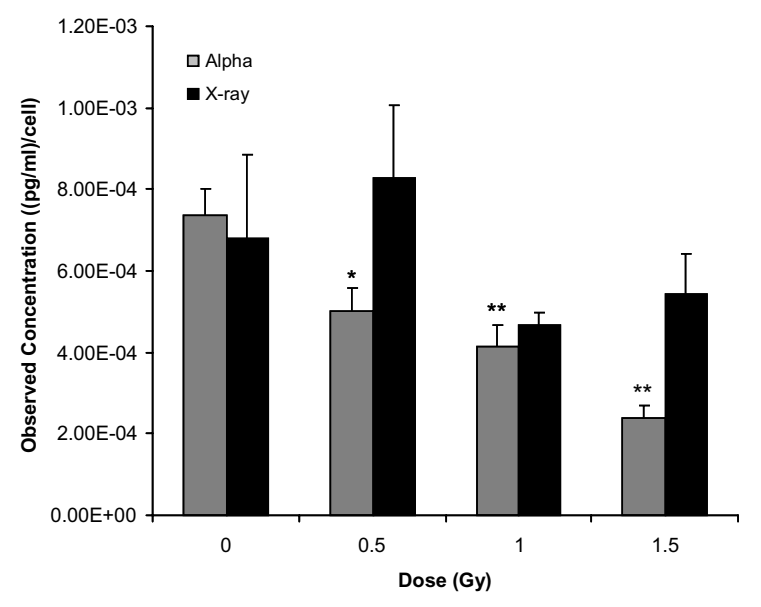

Figure 4. A549 cells were cultivated and exposed to $0 \mathrm{~Gy}-1.5 \mathrm{~Gy}$ of $\alpha$-particle or X-ray radiation. VEGF secretion levels were validated using an ELISA conducted per manufacturer's instructions. A highly significant $(\mathrm{p}<0.001)$ linear correlation was observed $\left(\mathrm{R}^{2}=0.9721\right)$. Statistical significances are presented ${ }^{*}$ represents $\mathrm{p}<0.05$, ** represents $\mathrm{p}<0.01$ based on $\mathrm{n}=4$ biological replicates. Bars are plotted as cell count corrected means \pm SD.

this is a very small percentage of the proteome, the proteins that were identified were abundant expressors (visible with coomassie blue staining) and therefore could potentially be robust biomarkers of $\alpha$-particle exposure. The specificity of this response needs to be verified, as these proteins may also be typically observed during any stress response. Many of the differentially expressed proteins were shown to be associated with important cellular activities including functions in cell motility/trafficking (annexin A2 and proteosome subunit alpha type-3) [5, 6], antioxidant production (peroxiredoxin-6) [7] and stress response processes (HSPA8) [8]. These results provide evidence to show that exposure to moderate level of $\alpha$-particle radiation can cause intracellular modifications of many protein components involved in cellular metabolic processes.

Cell culture supernatants were assessed for the expression of pro-inflammatory cytokines following cell irradiation to both $\alpha$ t-particles and X-rays. Distinct differences between responses were observed between the two radiation types. Of the 27 cytokines that were screened for differential expression, eight of these cytokines were $\alpha$-particle responsive. Only two, (Eotaxin and IFN- $\gamma$ ) were also shown to be modulated in the X-irradiated samples. Eotaxin is involved in the active recruitment of eosinophils by induction of their chemotaxis [9]. IFN- $\gamma$ can be produced as part of the innate immune response and has a diverse function in immuno-regulation, defence against viruses and tumour progression [10]. Of the $8 \alpha$-particle induced cytokines, VEGF was shown to be highly expressed and not secreted in Xirradiated samples. This cytokine has been shown to be involved in angiogenesis and over-expression of this protein is often associated with tumour formation and a number of disease states [11]. This particular cytokine was shown to be dose-responsive in $\alpha$-particle exposed samples and not statistically expressed in X-irradiated samples. Therefore, VEGF lends itself to be a strong candidate biomarker for $\alpha$-particle exposure. However, further validation of this data is presently being conducted in primary lymphocytes from normal human subjects.

\section{CONCLUSION}

Overall, these results indicate that the proteomic approach could represent a simple and useful tool for monitoring cellular radiation response in order to discover molecular biomarkers of radiation exposure. Future studies will focus efforts on identifying responses that are unique to $\alpha$-particles and as well 
examining low abundant expressers that may be present in the nuclear fractions of the total cell lysates. Such proteins may be more robust and represent distinct biomarkers of $\alpha$-particle radiation exposure.

\section{Acknowledgments}

Funding for this study was provided by the Health Canada Genomics Research and Development Initiative. The authors are grateful to Ngoc Vuong and Renaud Vincent for technical advice.

\section{References}

[1] Goldfarb A. and Litvinenko M., Death of a Dissident: The Poisoning of Alexander Litvinenko and the Return of the KGB (The Free Press, New York, 2007)

[2] Johns H.E. and Cunningham J.R., The Physics of Radiology, 4 ed. (Charles C. Thomas, Illinois, 1983)

[3] Jostes R.F., Mutat Res. 340 (1996) 125-139.

[4] Agostinelli S., Allison J., Amakoe K., Apostolakisa J., Araujoaj H., Arcel P., Asaig M., Axeni D., Banerjee S., ... Zschiesche D., Nuclear Instruments and Methods in Physics Research A 506 (2003) 250-303.

[5] Mai J., Finley R.L. Jr, Waisman D.M., Sloane B.F., J. Biol. Chem. 275 (2000) 12806-12812.

[6] Wang Y., Phelan S.A., Manevich Y., Feinstein S.I., Fisher., Am J Respir Cell Mol Biol. 34 (2006) 481-486.

[7] Touitou R., Richardson J., Bose S., Nakanishi M., Rivett J., Allday M.J., EMBO J. 20 (2001) 2367-2375.

[8] Prescott J., Coetzee G.A., Cancer Lett. 231 (2006) 12-19

[9] Gleich G.J., J Allergy Clin Immunol. 105 (2000) 651-663

[10] Schroder K., Hertzog P.J., Ravasi T., Hume D.A., J Leukoc Biol. 75 (2004) 163-89.

[11] Orpana A, Salven P. Leuk Lymphoma. 43 (2002) 219-24. 